\section{cef \\ ...leadless \\ pacing is \\ feasible and \\ relatively safe, \\ at least in the \\ short term}

\title{
Newly designed leadless pacemaker
}

Transvenous leads that deliver pacing therapy to the heart via a subcutaneous pacemaker cause complications in approximately $12.5 \%$ of patients implanted with the device. In a new study published in NEJM, the Micra Transcatheter Pacing Study Group describe a newly designed leadless transcatheter pacemaker that is as safe and efficacious as conventional transvenous systems.

Patients who met class I or II guideline-based indications for pacing were enrolled into the study, and implanted with the leadless pacemaker. The primary safety end point was freedom from system-related or procedure-related complications, whereas the primary efficacy end point was the composite of a low and stable pacing capture threshold at 6-month follow-up.

Of the 725 implantations performed, 719 (99.2\%) were successful. A Kaplan-Meier estimate calculation for freedom from major complications associated with the system or procedure was $96 \%$ at 6 months after implantation (95\% CI 93.9-97.3\%, $P<0.001$ when compared with safety performance goal of $83 \%$ ). A total of 28 major complications were identified among 25 patients, including cardiac injuries and injury at the groin puncture site. Of the 297 patients included in the primary efficacy analysis, $98.3 \%$ had an adequate 6-month pacing capture threshold.

The study investigators conclude that "the efficacy and safety outcomes among the patients in our study met both performance goals". In an accompanying editorial, Mark S. Link agrees that this study demonstrates "that leadless pacing is feasible and relatively safe, at least in the short term". However, he questions the value of this leadless device, given that "these newer devices can be used only for single-chamber ventricular pacing ... [and] will have limited usefulness in the treatment of the majority of pacemaker recipients".

Karina Huynh

ORIGINAL ARTICLE Reynolds, D. et al. A leadless intracardiac transcatheter pacing system. N. Engl. J.Med. doi:10.1056/NEJMoa1511643 
ERRATUM

Newly designed leadless pacemaker

Karina Huynh

Nat. Rev. Cardiol. 13, 5 (2016); doi: 10.1038/nrcardio.2015.187

In the version of the Research Highlight initially published online and in print, the text " $29.3 \%$ had an adequate 6-month pacing capture threshold" should have read " $98.3 \%$ had an adequate 6 -month pacing capture threshold". The error has been corrected for the HTML and PDF versions of the article. 